\title{
Avaliação do nível de satisfação dos moradores da Casa do Estudante Universitário I da Universidade Federal de Santa Maria com relação a aspectos estruturais e sociais
}

Evaluation of the satisfaction level of the students of the university student house I with concerning the structural and social aspects of the Federal University of Santa Maria

\author{
Maicon Facco', Marcos Antonio de Azevedo de Campos", Dagma Horbach"', \\ Marco Antonio Roberto Castagna Gonçalves ${ }^{1 v}$, Fernando Jesus Moreira Juniorv
}

\begin{abstract}
RESUMO
A Casa do Estudante Universitário, também conhecida como CEU, é um conjunto habitacional para estudantes da Universidade Federal de Santa Maria - UFSM e divide-se em duas unidades na cidade: uma localizada no centro da cidade, e outra no próprio campus. O presente trabalho tem por objetivo avaliar a satisfação dos moradores quanto aos diferentes aspectos estruturais e sociais, e propor possíveis melhorias. Para tal, foi aplicado um questionário elaborado com 13 perguntas entre estudantes selecionados por meio de uma amostragem por conglomerados referente aos apartamentos, com o sexo (masculino e feminino) sendo a variável de referência. Os integrantes do grupo do projeto aplicaram todos os questionários na Casa do Estudante Universitário I - CEU I, localizada no centro de Santa Maria - RS, num prazo de três horas de coleta, divididos em duplas e abordando cada apartamento amostrado e aplicando o questionário aos moradores do apartamento de forma individual. Foram, então, feitas inferências nos dados coletados através de técnicas multivariadas e análise descritiva. A partir de tais análises é sugerido que algumas reclamações sobre infraestrutura sejam consideradas em reuniões com os responsáveis pela direção da PRAE (Pró-Reitoria de Assuntos Estudantis) a fim de buscar soluções para os problemas descritos pelos estudantes.
\end{abstract}

Palavras-chave: Satisfação; Casa do estudante; Amostragem; Aspectos estruturais e sociais.

\begin{abstract}
The university student house, also known as CEU, is a housing developed for Federal University of Santa Maria (UFSM) students. There are two units of the housing in the city: one is located downtown and the other on campus. The present study aims to assess resident's satisfaction with different structural and social aspects, and propose possible improvements. To this end, a questionnaire designed with 13 questions was applied among students selected through cluster sampling for apartments, with gender (male and female) being the reference variable. The project group members applied all questionnaires at the University Student House I - CEU I, located in downtown of Santa Maria - RS, within three hours of collection, divided into pairs and approaching each apartment sampled and applying the questionnaire to residents of the apartment individually. Inferences were then made in the data collected through multivariate techniques and descriptive analysis. From these analyzes it is suggested that some infrastructure complaints be considered in meetings with PRAE's (Pró-Reitoria de Assuntos Estudantis) senior management to seek solutions to the problems described by the students.
\end{abstract}

Keywords: Satisfaction; Student house; Sampling; Structural and social aspects.

\footnotetext{
I Universidade Federal de Santa Maria, Santa Maria, Brasil. E-mail: maicon_facco@yahoo.com.br.

" Universidade Federal de Santa Maria, Santa Maria, Brasil. E-mail: marcos130694@gmail.com.

II' Universidade Federal de Santa Maria, Santa Maria, Brasil. E-mail: dagmahorbach@hotmail.com.

IV Universidade Federal de Santa Maria, Santa Maria, Brasil. E-mail: marcoarcg@gmail.com.

v Universidade Federal de Santa Maria, Santa Maria, Brasil. E-mail: fmjunior777@yahoo.com.br.
} 


\section{INTRODUÇÃO}

A Casa do Estudante Universitário (CEU) é um espaço de moradia e também um recurso institucional que é disponibilizada para o estudante com o objetivo de mantêlo próximo à sua realidade acadêmica quando este não reside na cidade da própria instituição. As Casas de Estudantes são caracterizadas por diversas situações que implicam em variáveis semelhantes, como população jovem, separação das famílias, vida coletiva, projeto de carreira, dentre outras, incluindo todas as implicações que elas possam representar. Nelas, a convivência cotidiana favorece a constituição de um grupo informal de redes e de relações importantes, que passam a ter suas próprias regras de conduta e relacionamentos. Além disso, a conquista de uma vaga na universidade pública pode ser invalidada pela dificuldade em manter-se na moradia estudantil, pois sabe-se que os jovens dependem de uma estrutura de apoio que inclui alimentação, moradia, assistência médica e odontológica, transporte e recursos para manutenção durante o curso (FONAPRACE, 2004).

A Casa do Estudante Universitário da Universidade Federal de Santa Maria (UFSM) possui duas unidades: uma localizada no centro de Santa Maria (CEU I), e outra localizada no campus sede da UFSM. Conforme breve histórico do site da UFSM (http://w3.ufsm.br/ceu1/historico.html), a Casa do Estudante Universitário I (CEU I) teve sua inauguração em meados de outubro de 1963, com 7 pavimentos destinados à moradia estudantil e às instalações do Diretório Central do Estudante.

A Casa do Estudante é uma unidade que compõe a Política de Assistência Estudantil, e destina-se servir de moradia aos estudantes universitários graduandos e aos estudantes dos cursos técnicos do Colégio Politécnico e Colégio Técnico Industrial, não residentes em Santa Maria, regularmente matriculados na UFSM e com benefício sócio econômico - BSE aprovado pela Pró Reitoria de Assuntos Estudantis (PRAE).

Atualmente, de acordo com site próprio da Casa de Estudante I, a mesma é composta por 62 apartamentos, dos quais 56 possuem capacidade para abrigar 4 estudantes e 6 apartamentos para abrigar 1 estudante, além de 7 salas direcionadas a estudos. Os quartos são classificados em mistos, femininos e masculinos, oferece gratuitamente moradia, no máximo, a 230 acadêmicos. Possui no hall de entrada 
biblioteca, coordenação do CEU I, lan-house, sala de estar com televisão, laboratório de informática (LABINFO) e Diretório Central do Estudante (DCE). Oferece também área de lazer no subsolo com quadra de vôlei, salão de festas (Catacumba) e churrasqueira.

Há diversos artigos que estudaram moradores de casas estudantis universitárias e que apresentaram resultados interessantes que serviram como motivação para esse estudo, tais como Martins (2002), que investigou o processo de individualização das estudantes residentes durante as décadas de 1950 e 1960 na Universidade Federal do Paraná a partir de entrevistas com ex-moradoras e documentos que serviram de fonte, ou Laranjo (2003) e Laranjo e Soares (2006), que investigaram como se desenvolvem os processos de socialização entre os estudantes residentes da moradia estudantil de uma universidade do estado de São Paulo, além de buscarem apreender o discurso dos moradores sobre o consumo de drogas no ambiente da moradia considerando dificuldades que ocorrem nos processos de adaptação à universidade e à moradia estudantil. Paiva e Mendes (2002), averiguaram quais eram os comportamentos e as percepções sobre territorialidade e privacidade dos moradores da Casa do Estudante Universitário da Universidade de Brasília através da psicologia ambiental. Araújo e Morgado (2007) investigaram como o conjunto de ações de assistência estudantil impacta positivamente os estudantes, incluindo aí a condição de ser residente em uma moradia estudantil, objetivando avaliar a influência da assistência estudantil sobre os estudantes e não a assistência propriamente dita. Dentre eles também há Garrido (2013), que apontou diferentes focos, agrupados em três categorias: o estudante morador, a moradia estudantil e as ações da assistência estudantil e constatou que embora haja ampla produção científica estrangeira voltada ao estudo das moradias universitárias, a nacional ainda é escassa e a visão da moradia como espaço de formação está pouco presente. Garrido (2015) verificou que há uma necessidade de investimentos nesses espaços, especialmente no que tange aos aspectos estruturais, com vistas a diminuir as dificuldades enfrentadas pelos moradores.

O objetivo geral deste artigo é avaliar o nível de satisfação dos moradores da casa do estudante universitário de localização central (CEU I) da Universidade Federal de Santa Maria com relação aos diferentes aspectos estruturais e sociais. Foram 
analisados os perfis dos atuais moradores da casa do estudante universitário levandose em consideração os sexos, as idades, os cursos e o tempo em que residem na CEU I. Também foi levado em consideração o nível de satisfação dos diversos aspectos abordados na CEU I separados por sexo, além da realização do agrupamento das variáveis em fatores de modo a gerar uma interpretação prática dos principais aspectos sociais e estruturais avaliados nesta pesquisa.

Até o momento que esse trabalho foi proposto, não havia sido realizada nenhuma pesquisa de satisfação sobre a casa do estudante da UFSM. Diante disso, vimos a necessidade de dar a oportunidade para que os moradores pudessem expor sua opinião sobre o lugar em que residem, com a finalidade de analisar e verificar as oportunidades de melhoria, buscando apresentar uma proposta de direcionamento para possíveis mudanças que visem contemplar o bem-estar dos universitários residentes do referido conjunto habitacional.

\section{METODOLOGIA}

As informações obtidas foram sobre o perfil do estudante e a satisfação do mesmo com relação aos aspectos estruturais e sociais dentro da moradia estudantil da universidade localizada no centro da cidade. Estas informações foram coletadas através de questionário a partir de abordagem pessoal, fazendo-se uso da escala Likert para avaliar o nível de satisfação. Foram feitas perguntas objetivas, constando de 13 perguntas, com tempo total médio de 5 minutos de aplicação.

O plano de amostragem foi amostragem por conglomerados (AC) em 1 estágio a partir de uma amostra de 54 apartamentos (conglomerados), considerando uma margem de erro de $5 \%$ e nível de $95 \%$ de confiança. Definiu-se como população alvo os estudantes residentes da Casa do Estudante Universitário I - CEU I. Os estudantes do CEU II não foram selecionados para a pesquisa pois não foram encontradas informações de controle sobre quaisquer variáveis relacionadas aos mesmos, sendo estas informações buscadas exaustivamente. Considerou-se como critério de inclusão todos os estudantes que estiveram presentes nos apartamentos do CEU I no horário em que ocorreu a aplicação dos questionários. Foram excluídos da pesquisa somente estudantes que se recusaram a responder o questionário, independente da motivação 
pessoal para tal. Foi realizada uma análise descritiva dos dados tabulados, análises gráficas e de tabelas de frequências e análise fatorial exploratória, assim como o cálculo do alfa de Cronbach. O software R Core Team (2018), assim como o Excel 2016, foram utilizados para as análises estatísticas e para a análise fatorial.

Conforme King (1986) em um modelo de análise fatorial, há muitas variáveis observadas e o objetivo é gerar fatores subjacentes que não são observados, onde os fatores, segundo Hair et al (2009), são combinações lineares das variáveis originais. A redução de uma grande quantidade de variáveis observadas em um número menor de fatores é o principal objetivo das diferentes técnicas multivariadas, incluindo a utilizada neste trabalho, denominada "Análise Fatorial pelo Método dos Componentes Principais".

Ainda segundo Hair et al, (2009), uma forte fundamentação conceitual é necessária para embasar a suposição de que existe uma estrutura antes de ser realizada a análise fatorial. A primeira ação durante a implementação de uma análise fatorial é analisar se os dados podem ser submetidos ao processo de análise fatorial (PASQUALI, 1998), ou seja, observar se a matriz de dados é passível de fatoração. É necessário realizar o teste de esfericidade de Bartlett para identificar se há correlações suficientes para se realizar a análise. Medidas de valores de adequação da amostra devem ser maiores do que 0,50 tanto para o teste geral quanto para cada variável individualmente. As variáveis que possuírem valor menor do que 0,50 devem ser omitidas uma por vez, sendo sempre a de menor valor a primeira a ser removida. Quando o nível de significância $p<0,05$ significa que a matriz é fatorável (TABACHNICK \& FIDELL, 2007), rejeitando a hipótese nula de que a matriz de dados é similar a uma matriz identidade, ou seja, significa que a matriz não possui valores na diagonal principal próximos de 1 e os demais elementos iguais a 0 , isto é, não se assemelha a uma matriz de variância e covariância onde as variáveis não possuem correlação alguma.

Outro método de avaliação necessário é o índice de Kaiser-Meyer-Olkin (KMO), um teste estatístico que sugere a proporção de variância dos itens que pode estar sendo explicada por uma variável latente (LORENZO-SEVA, TIMMERMAN \& KIERS, 2011). 
Este índice dá um indicativo da adequação da análise fatorial para o conjunto de dados. O KMO é calculado por meio do quadrado das correlações totais dividido pelo quadrado das correlações parciais, das variáveis analisadas (FIELD, 2009). Seu valor pode variar de zero a um. Os valores que são iguais ou próximos a zero indicam que a soma das correlações parciais dos itens avaliados é bastante alta em relação à soma das correlações totais, indicando assim que a análise fatorial possivelmente será inapropriada (PASQUALI, 1999). Como regra, valores do KMO menores que 0,5 são considerados inaceitáveis, valores entre 0,5 e 0,7 são considerados medíocres, valores entre 0,7 e 0,8 são considerados bons, valores maiores que 0,8 e 0,9 são considerados ótimos e excelentes, respectivamente (HUTCHESON \& SOFRONIOU, 1999).

Os resultados estão disponíveis no link https://drive.google.com/open?id=1lc1 uEQtShhQo2FZfMmfRmlkj-IzCv69- para que qualquer órgão ou setor vinculado à UFSM que tenha interesse possa otimizar e formular propostas para os resultados obtidos.

\section{RESULTADOS E DISCUSSÃO}

\subsection{Análise Descritiva}

Primeiramente, foi calculado o alfa de Cronbach e este apresentou como resultado 0,7101, indicando que o questionário aplicado possui uma consistência interna adequada.

Com base nas análises descritivas pode-se observar que o perfil dos moradores da CEU I é bastante heterogêneo. A amostra foi constituída por 74 estudantes, sendo 38 mulheres e 36 homens, de 54 apartamentos e de 30 cursos distintos. Na Tabela 1, assim como na Figura 1, observa-se que alguns cursos tiveram maior ocorrência na amostra, dentre eles destaca-se o curso de Direito, com 13 ocorrências (aproximadamente 18\% da amostra), o curso de Relações Internacionais, com 8 ocorrências (aproximadamente 11\% da amostra) e o curso de Ciências Contábeis, com 6 ocorrências (aproximadamente $8 \%$ da amostra). Além dos cursos em destaque, foram encontrados 16 cursos com apenas uma ocorrência na amostra. 
Tabela 1 - Frequência de moradores da CEU I separados por curso

\begin{tabular}{lcc}
\hline \multirow{2}{*}{ Curso } & \multicolumn{2}{c}{ Frequência } \\
\cline { 2 - 3 } & Absoluta & Relativa \\
\hline Ciências contábeis & 6 & $8 \%$ \\
Dança & 2 & $3 \%$ \\
Direito & 13 & $17 \%$ \\
Economia & 4 & $5 \%$ \\
Educação Especial & 2 & $3 \%$ \\
Educação Física & 3 & $4 \%$ \\
Fonoaudiologia & 3 & $4 \%$ \\
Letras-Espanhol & 2 & $3 \%$ \\
Letras-Português & 2 & $3 \%$ \\
Odontologia & 5 & $7 \%$ \\
Pedagogia & 3 & $4 \%$ \\
Relações internacionais & 8 & $11 \%$ \\
Tecnologia em alimentos & 2 & $3 \%$ \\
Zootecnia & 3 & $4 \%$ \\
Outros & 16 & $21 \%$ \\
\hline Total & 74 & $100 \%$ \\
\hline
\end{tabular}

Fonte: Dados da pesquisa.

Figura 1 - Moradores da CEU I separados por curso

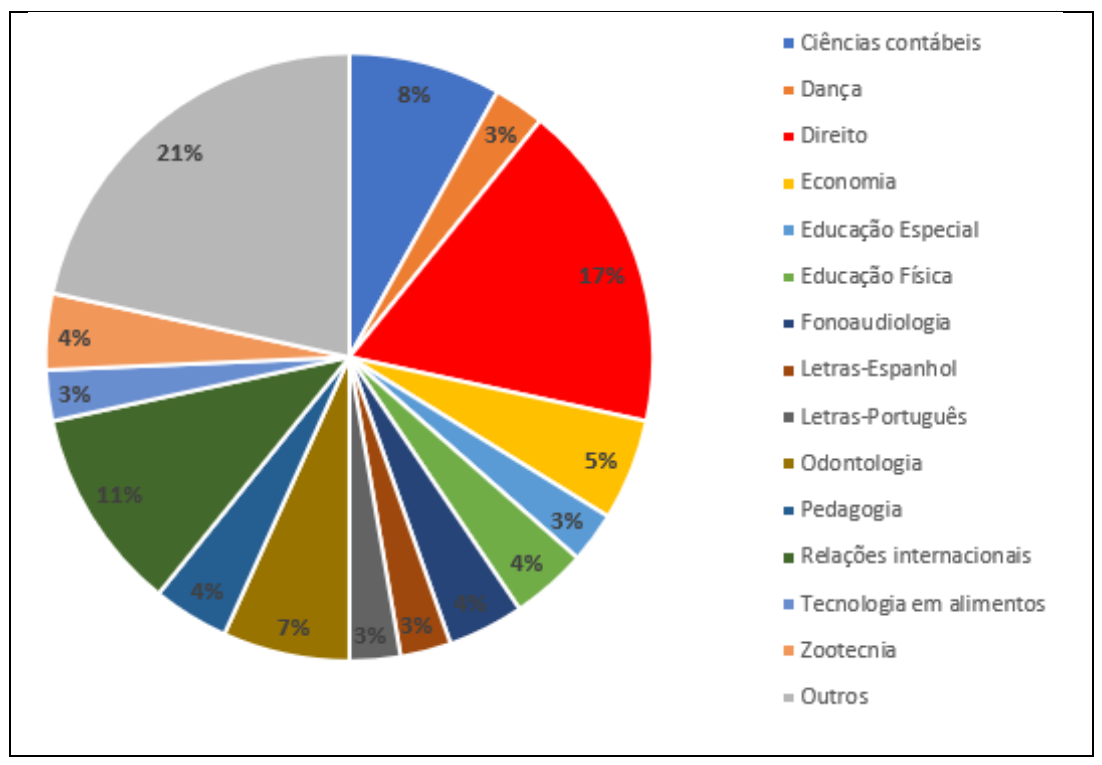

Fonte: Os autores.

A partir da Tabela 2, pode-se observar que a maior parte dos moradores estão morando na CEU I entre 1 e 3 anos, sendo que a maior parte dos moradores do sexo feminino estão até 2 anos na CEU I, enquanto que a maior parte dos moradores do sexo masculino estão a mais de 2 anos na CEU I. 
Tabela 2 - Moradores da CEU 1 separados por sexo e tempo de permanência

\begin{tabular}{lrrrrrr}
\hline \multirow{2}{*}{ Sexo } & \multicolumn{5}{c}{ Tempo de permanência } & \\
\cline { 2 - 6 } & até 1 ano & mais de 1 a 2 anos & mais de 2 a 3 anos & mais de 3 anos & Total \\
\hline Feminino & 6 & 15 & 11 & 6 & 38 \\
Masculino & 6 & 8 & 12 & 10 & 36 \\
\hline Total & 12 & 23 & 23 & 16 & 74 \\
\hline
\end{tabular}

Fonte: Os autores.

Como pode ser observado na Tabela 3, maior parte dos moradores da CEU I têm entre 18 e 25 anos, sendo que a maior parte dos moradores do sexo feminino está entre 18 e 21, enquanto o sexo masculino está entre 22 e 25 anos.

Para os diferentes níveis de satisfação foram avaliados os aspectos acessibilidade, segurança, conforto, privacidade, limpeza interna e externa, áreas de lazer e estudo e banheiros.

Tabela 3 - Moradores da CEU I separados por faixa etária e sexo

\begin{tabular}{lrrr}
\hline \multirow{2}{*}{ Faixa etária } & \multicolumn{2}{c}{ Sexo } & \multirow{2}{*}{ Total } \\
\cline { 2 - 4 } & Feminino & Masculino & \\
\hline $18-21$ & 28 & 13 & 41 \\
$22-25$ & 9 & 17 & 26 \\
$26-29$ & 1 & 3 & 4 \\
$30-33$ & 0 & 2 & 2 \\
mais de 34 & 0 & 1 & 1 \\
\hline Total & 38 & 36 & 74 \\
\hline
\end{tabular}

Fonte: Os autores.

Para a variável Acessibilidade, Tabela 4, observa-se que tanto as pessoas de sexo feminino quanto masculino tem opiniões semelhantes, a maior parte está muito satisfeita com relação à acessibilidade e 11 estudantes disseram estar extremamente satisfeitos. Mais de $70 \%$ está satisfeito e isso pode ser explicado por haver dois elevadores no local, além de rampa de acesso na entrada da CEU I.

Tabela 4 - Satisfação com relação a acessibilidade da CEU I separadas por sexo

\begin{tabular}{crrrrrr}
\hline \multirow{2}{*}{ Sexo } & \multicolumn{5}{c}{ Acessibilidade } & \\
\cline { 2 - 7 } & Insatisfeito & Pouco satisfeito & Satisfeito & Muito satisfeito & Extremamente satisfeito & \\
\hline Feminino & 4 & 3 & 7 & 19 & 5 & 38 \\
Masculino & 4 & 5 & 10 & 11 & 6 & 36 \\
\hline Total & 8 & 8 & 17 & 30 & 11 & 74 \\
\hline
\end{tabular}

Fonte: Os autores. 
Com relação à segurança, Tabela 5, a maior parte das mulheres disse estar satisfeita, enquanto os homens não demonstraram tanta satisfação; muitos disseram estar pouco satisfeitos e 2 ainda responderam estar insatisfeitos.

Tabela 5 - Satisfação com relação a segurança da CEU I separadas por sexo

\begin{tabular}{lrrrrrr}
\hline \multirow{2}{*}{ Sexo } & \multicolumn{5}{c}{ Segurança } & Total \\
\cline { 2 - 7 } & Insatisfeito & Pouco satisfeito & Satisfeito & Muito satisfeito & Extremamente satisfeito & \\
\hline Feminino & 0 & 6 & 12 & 17 & 3 & 38 \\
Masculino & 2 & 12 & 9 & 10 & 3 & 36 \\
\hline Total & 2 & 18 & 21 & 27 & 6 & 74 \\
\hline
\end{tabular}

Fonte: Os autores.

Para Conforto, Tabela 6, pode-se observar que tanto as pessoas de sexo feminino quanto masculino tem opiniões semelhantes. A maior parte está muito satisfeita com relação ao conforto e 6 estudantes disseram estar extremamente satisfeitos. Quase $80 \%$ está satisfeito, muito ou extremamente satisfeito.

Tabela 6 - Satisfação com relação a conforto da CEU I separadas por sexo

\begin{tabular}{|c|c|c|c|c|c|c|}
\hline \multirow{2}{*}{ Sexo } & \multicolumn{5}{|c|}{ Conforto } & \multirow{2}{*}{ Total } \\
\hline & Insatisfeito & Pouco satisfeito & Satisfeito & Muito satisfeito & Extremamente satisfeito & \\
\hline Feminino & 2 & 4 & 14 & 14 & 4 & 38 \\
\hline Masculino & 4 & 5 & 11 & 14 & 2 & 36 \\
\hline Total & 6 & 9 & 25 & 28 & 6 & 74 \\
\hline
\end{tabular}

Fonte: Os autores.

Já com relação à privacidade, Tabela 7, houve um menor nível de satisfação. A maior parte dos homens se diz pouco satisfeito, enquanto as mulheres demonstraram maior satisfação, sendo a maioria satisfeita ou muito satisfeita. Porém, no geral, a maioria dos estudantes estão, no máximo, satisfeitos. Isso pode ser atribuído ao fato de que há uma insatisfação dos moradores em relação à infraestrutura do prédio, pois os banheiros são fora dos apartamentos, e dentro dos apartamentos as paredes que separam os quartos são de plástico e não alcançam o teto. Ainda, os varais para estender as roupas são localizados nos corredores. 
Tabela 7 - Satisfação com relação a privacidade da CEU I separadas por sexo

\begin{tabular}{|c|c|c|c|c|c|c|}
\hline \multirow{2}{*}{ Sexo } & \multicolumn{5}{|c|}{ Privacidade } & \multirow{2}{*}{ Total } \\
\hline & Insatisfeito & Pouco satisfeito & Satisfeito & Muito satisfeito & Extremamente satisfeito & \\
\hline Feminino & 8 & 6 & 12 & 12 & 0 & 38 \\
\hline Masculino & 6 & 14 & 12 & 4 & 0 & 36 \\
\hline Total & 14 & 20 & 24 & 16 & 0 & 74 \\
\hline
\end{tabular}

Fonte: Os autores.

Para limpeza interna e limpeza externa (Tabela 8 e 9, respectivamente), podemos observar que as pessoas demonstraram maior satisfação com relação à limpeza interna do que com a externa, mas em ambos os casos o nível de satisfação foi em maior parte positivo, e ainda houve maior satisfação por parte das mulheres, porém não sendo uma diferença significativa.

Tabela 8 - Satisfação com relação a limpeza interna da CEU I separadas por sexo

\begin{tabular}{lccccccc}
\hline \multirow{2}{*}{ Sexo } & \multicolumn{6}{c}{ Privacidade } & \multirow{2}{*}{ Total } \\
\cline { 2 - 7 } & Insatisfeito & Pouco satisfeito & Satisfeito & Muito satisfeito & Extremamente satisfeito & \\
\hline Feminino & 0 & 5 & 11 & 17 & 5 & 38 \\
Masculino & 2 & 2 & 13 & 14 & 5 & 36 \\
\hline Total & 2 & 7 & 24 & 31 & 10 & 74 \\
\hline
\end{tabular}

Fonte: Os autores.

Tabela 9 - Satisfação com relação a limpeza externa da CEU I separadas por sexo

\begin{tabular}{lccccccc}
\hline \multirow{2}{*}{ Sexo } & \multicolumn{6}{c}{ Limpeza Externa } & \multirow{2}{*}{ Total } \\
\cline { 2 - 7 } & Insatisfeito & Pouco satisfeito & Satisfeito & Muito satisfeito & Extremamente satisfeito & \\
\hline Feminino & 2 & 4 & 12 & 16 & 4 & 38 \\
Masculino & 2 & 10 & 11 & 10 & 3 & 36 \\
\hline Total & 4 & 14 & 23 & 26 & 7 & 74 \\
\hline
\end{tabular}

Fonte: Os autores.

Com relação às áreas de lazer e estudo (Tabelas 10 e 11, respectivamente), a maior parte dos estudantes responderam de forma neutra, demonstrando estarem apenas satisfeitos, porém maior parte dos homens responderam estar muito satisfeitos com relação à área de lazer e apenas 2 insatisfeitos, enquanto que quase 10\% disseram estar insatisfeitos com relação à área de estudo. Já as mulheres mantiveram neutralidade em ambos os casos, respondendo estarem apenas satisfeitas. 
Tabela 10 - Satisfação com relação a área de lazer da CEU I separadas por sexo

\begin{tabular}{|c|c|c|c|c|c|c|}
\hline \multirow{2}{*}{ Sexo } & \multicolumn{5}{|c|}{ Área de Lazer } & \multirow{2}{*}{ Total } \\
\hline & Insatisfeito & Pouco satisfeito & Satisfeito & Muito satisfeito & Extremamente satisfeito & \\
\hline Feminino & 2 & 5 & 17 & 10 & 4 & 38 \\
\hline Masculino & 2 & 9 & 9 & 13 & 3 & 36 \\
\hline Total & 4 & 14 & 26 & 23 & 7 & 74 \\
\hline
\end{tabular}

Fonte: Os autores.

Tabela 11 - Satisfação com relação a área de estudo da CEU 1 separadas por sexo

\begin{tabular}{|c|c|c|c|c|c|c|}
\hline \multirow{2}{*}{ Sexo } & \multicolumn{5}{|c|}{ Área de Estudo } & \multirow{2}{*}{ Total } \\
\hline & Insatisfeito & Pouco satisfeito & Satisfeito & Muito satisfeito & Extremamente satisfeito & \\
\hline Feminino & 1 & 6 & 17 & 10 & 4 & 38 \\
\hline Masculino & 7 & 6 & 12 & 8 & 3 & 36 \\
\hline Total & 8 & 12 & 29 & 18 & 7 & 74 \\
\hline
\end{tabular}

Fonte: Os autores.

Já com relação aos banheiros, Tabela 12, houve um menor nível de satisfação: a maior parte dos estudantes disseram estar, no máximo, apenas satisfeitos, sendo a maioria satisfeita ou pouco satisfeita. Para ambos os sexos, o nível de satisfação foi semelhante com relação aos banheiros. E apenas um homem disse estar extremamente satisfeito.

Tabela 12 - Satisfação com relação ao banheiro da CEU I separadas por sexo

\begin{tabular}{|c|c|c|c|c|c|c|}
\hline \multirow{2}{*}{ Sexo } & \multicolumn{5}{|c|}{ Área de Estudo } & \multirow{2}{*}{ Total } \\
\hline & Insatisfeito & Pouco satisfeito & Satisfeito & Muito satisfeito & Extremamente satisfeito & \\
\hline Feminino & 4 & 11 & 14 & 9 & 0 & 38 \\
\hline Masculino & 5 & 10 & 12 & 8 & 1 & 36 \\
\hline Total & 9 & 21 & 26 & 17 & 1 & 74 \\
\hline
\end{tabular}

Fonte: Os autores.

\subsection{Análise Fatorial}

No presente estudo, o resultado para o teste de Bartlett resultou em um p-valor $<0,00001$, significando que a hipótese nula foi rejeitada, isto é, a matriz de dados das respostas dos estudantes no questionário aplicado não se assemelha a uma matriz identidade, indicando a presença de correlação entre suas respostas quanto a cada variável. 
Ainda, para o teste KMO, o valor do índice calculado foi de 0,66 , indicando uma baixa (porém aceitável) adequação da análise fatorial com relação aos dados dos questionários dos respondentes.

A Tabela 13 mostra as variáveis e suas respectivas cargas fatoriais, sendo agrupadas nos fatores aquelas que possuem maior carga fatorial e superior a 0,4.

Tabela 13 - Valores das cargas fatoriais das variáveis rotacionadas associadas a cada um dos componentes

\begin{tabular}{lccccc}
\hline \multirow{2}{*}{ Variáveis } & \multicolumn{6}{c}{ Componentes } \\
\cline { 2 - 6 } & 1 & 2 & 3 & 4 & 5 \\
\hline Banheiro & 0,68 & 0,26 & 0,34 & $-0,11$ & 0,14 \\
Segurança & 0,62 & $-0,34$ & $-0,14$ & $-0,21$ & 0,15 \\
Limpeza Interna & 0,62 & $-0,02$ & 0,47 & 0,37 & 0,02 \\
Limpeza Externa & 0,59 & $-0,21$ & 0,05 & 0,40 & 0,20 \\
Sexo & $-0,19$ & 0,74 & $-0,02$ & 0,00 & 0,02 \\
Idade & 0,01 & 0,74 & $-0,10$ & $-0,22$ & $-0,12$ \\
Tempo de Permanência & 0,35 & 0,53 & $-0,29$ & 0,30 & $-0,15$ \\
Privacidade & 0,14 & $-0,13$ & 0,90 & $-0,01$ & $-0,03$ \\
Conforto & 0,03 & $-0,07$ & 0,81 & 0,06 & 0,30 \\
Acessibilidade & $-0,01$ & $-0,08$ & 0,03 & 0,90 & 0,06 \\
Área de Lazer & 0,15 & 0,10 & 0,12 & 0,19 & 0,82 \\
Área de Estudo & 0,12 & $-0,26$ & 0,10 & $-0,09$ & 0,77 \\
\hline Fonyyyyyyyy
\end{tabular}

Fonte: Os autores.

Conforme se pode notar, o componente 1 agrupou as variáveis Segurança, Limpeza Interna, Limpeza Externa e Banheiro, variáveis estas responsáveis pela caracterização da moradia estudantil. No fator 2, o que se nota é que apresentaram cargas fatoriais elevadas as variáveis relacionadas às questões relativas a informações de perfil dos moradores da Casa Do Estudante Universitário I, tais como Sexo, Idade e Tempo de Permanência. Nesse fator, observa-se que as variáveis que mostram as informações de perfil dos moradores foram agrupadas de forma que duas delas (Sexo e Idade) apresentaram carga fatorial superior a 0,7. Já no fator 3, as variáveis com maior carga fatorial são indicadoras da importância do Conforto e da Privacidade para o estudante. Para o fator 4 foi considerado como um único grupo a variável relacionada às condições referentes à acessibilidade dentro do CEU I. No fator 5 foram agrupadas as variáveis referentes às áreas de estudo e lazer, consideradas de grande importância no dia-a-dia dos acadêmicos. 
A análise dos fatores extraídos da amostra permite concluir que uma adequada alocação foi feita, agrupando as variáveis em 5 grupos distintos: o primeiro tratando das condições da conservação e segurança da moradia estudantil, o segundo referente às características pessoais do morador da Casa do Estudante Universitário, o terceiro relacionado ao bem-estar do morador, o quarto referente à qualidade de acesso e o quinto grupo baseado no espaço direcionado para o estudante realizar suas atividades, sejam elas pessoais ou acadêmicas.

Além disso, todas as variáveis foram alocadas em algum fator. Caso alguma carga fatorial fosse inferior a 0,4 em todos os fatores, significaria que tal variável não obteve desempenho estatisticamente satisfatório na aplicação do questionário e, portanto, poderia ser descartada caso o mesmo questionário fosse replicado. Em suma, o Fator 1 (Conforto e Privacidade) explicou 16\% da variabilidade total, o Fator 2 (Segurança, Limpeza Interna, Limpeza Externa e Banheiro) explicou 16\%, o Fator 3 (Sexo, Idade e Tempo), 14\%, o Fator 4 (Lazer e Estudo), 12\% e o Fator 5 (Acessibilidade), 11\%, ou seja, obteve-se um total de $69 \%$ de variabilidade explicada.

\section{CONCLUSÕES}

Com base nos resultados obtidos na pesquisa, ficou evidente que na realização da análise fatorial houve uma separação coerente em cinco fatores, onde cada um engloba diferentes aspectos relacionados à satisfação dos estudantes. Variáveis como Segurança, Limpeza Interna, Limpeza Externa e Banheiro compõem um primeiro fator; Sexo, Idade e Tempo de Permanência outro fator; Conforto e Privacidade, outro; Acessibilidade à moradia estudantil foi alocada em um fator isolado e por último foram agrupadas as variáveis Área de Lazer e Área de Estudo. A análise fatorial considerou que nenhuma variável do questionário deve ser excluída em caso de replicação do mesmo.

A partir das análises estatísticas realizadas, é possível afirmar que a maior aceitação por parte dos moradores é com relação à acessibilidade (componente do fator 4 na análise fatorial), e que grande parte dos moradores se sente satisfeita com relação aos aspectos de limpeza interna e externa, segurança e conforto. 
É importante destacar que um dos quesitos que geram insatisfação dos moradores é com relação aos banheiros. Outro ponto de atenção é em relação à privacidade, onde a maioria dos moradores disseram estar no máximo satisfeitos e isso se deve ao fato de que maior parte dos quartos são coletivos. Com relação aos banheiros do CEU I e à privacidade, é importante ressaltar que a grande insatisfação dos moradores é em relação à infraestrutura do prédio, pois os banheiros são fora dos apartamentos, e que dentro dos apartamentos as paredes que separam os quartos são de plástico e não alcançam o teto. Ainda, os varais para estender as roupas são localizados nos corredores. Sendo assim, sugere-se que a reclamação seja considerada em reuniões de departamentos com os responsáveis pela direção da PRAE, para que seja estudada uma mudança na infraestrutura e/ou organização. 


\section{REFERÊNCIAS}

DE LIMA, Carla Patrícia Novaes. A ARTE DA PARTICIPAÇÃO E A PARTICIPAÇÃO PELA ARTE: UMA EXPERIÊNCIA NAS CASAS DE ESTUDANTES UNIVERSITÁRIAS DA UFPE [1].

FIELD, Andy. Discopering Statistics Using SPSS, Thrid Edition. 2009.

FONAPRACE. Perfil Socioeconômico e Cultural dos Estudantes de Graduação das Instituições Federais de Ensino Superior: Relatório Final da Pesquisa. Fórum Nacional de Pró-Reitores de Assuntos Comunitários e Estudantis. FONAPRACE, Brasília. 2004

GARRIDO, Edleusa Nery et al. A moradia estudantil universitária como tema na produção científica nacional. Psicologia Escolar e Educacional, 2013.

GARRIDO, Edleusa Nery. A experiência da moradia estudantil universitária: impactos sobre seus Moradores. Psicologia: ciência e profissão, v. 35, n. 3, p. 726-739, 2015.

HAIR, Joseph F. et al. Análise multivariada de dados. Bookman Editora, 2009.

HUTCHESON, Graeme D.; SOFRONIOU, Nick. The multivariate social scientist: Introductory statistics using generalized linear models. Sage, 1999.

KING, Gary. How not to lie with statistics: Avoiding common mistakes in quantitative political science. American Journal of Political Science, p. 666-687, 1986.

LARANJO, Thais Helena Mourão. 0 Crusp: processos de socialização e consumo de drogas. 2003. Tese de Doutorado. Universidade de São Paulo.

LARANJO, Thais Helena Mourão; SOARES, Cássia Baldini. Moradia universitária: processos de socialização e consumo de drogas. Revista de Saúde Pública, v. 40, p. 1027-1034, 2006.

LORENZO-SEVA, Urbano; TIMMERMAN, Marieke E.; KIERS, Henk AL. The Hull method for selecting the number of common factors. Multivariate behavioral research, v. 46, n. 2, p. 340-364, 2011.

MARTINS, Ana Paula Vosne. Um lar em terra estranha: a casa da estudante universitária em Curitiba e o processo de individualização feminina nas décadas de 1950 e 1960. 2002.

MORGADO, Maria Aparecida. Educação e Juventude: um panorama da assistência estudantil e de seus usuários na UFMT (Cuiabá) ARAÚJO, Denise Pereira de. Universidade Federal de Mato Grosso. 
PAIVA, D.; MENDES, G. Onde se pode ficar nu: territorialidade e privacidade na casa do estudante universitário da UNB. Textos do laboratório de psicologia ambiental, v. 6, 2002.

PASQUALI, Luís. Análise fatorial: um manual teórico-prático. Brasília: Editora da UnB, no prelo, 1998.

R Core Team (2018). R: A language and environment for statistical computing. R Foundation for Statistical Computing, Vienna, Austria. URL https://www.Rproject.org/.

TABACHNICK, Barbara G.; FIDELL, Linda S.; ULLMAN, Jodie B. Using multivariate statistics. Boston, MA: Pearson, 2007.

UFSM. Casa do estudante universitário - CEU I, c2018. Histórico. Disponível em: http://w3.ufsm.br/ceu1/historico.html. Acesso em: 16 de out. de 2018. 